\title{
Intestinal Barrier Dysfunction: The Primary Driver of IBD?
}

\author{
Pieter Hindryckx and Debby Laukens \\ Ghent University \\ Belgium
}

\section{Introduction}

The healthy gastrointestinal tract is functionally maintained by an epithelial barrier, a monocellular layer that acts as a critical interface between the "outside" lumen and host tissues. This selectively permeable barrier controls the equilibrium between tolerance and immunity to microbes and non-self antigens. It is physically composed of epithelial cells linked through tight junctions, and it is reinforced by a mucus layer and the secretion of antimicrobial peptides such as defensins, cathelicidins and lysozymes. Intestinal epithelial cells are also responsible for the transport of water and nutrients while simultaneously preventing the uptake of noxious agents and luminal flora. Pathogens are selectively eradicated and should therefore be distinguished from the commensal flora to elicit a balanced inflammatory response. This capacity is tightly governed by pathogen recognition receptors, such as NOD proteins and the Toll-like receptors (TLRs). To avoid immunologic hyper-responsiveness against harmless intra-luminal food and bacterial antigens, the selective transport of small quantities of these antigens takes place by dendritic cells and $\mathrm{M}$ cells in Peyer's patches, leading to oral tolerance.

Several defects related to intestinal barrier function have been found in patients with inflammatory bowel disease (IBD), but for many, it remains to be clarified whether these are primary defects or secondary bystander effects of the inflammatory state. Nevertheless, evidence suggests that a "leaky gut" is an early and possibly primary defect in IBD pathogenesis. It has been demonstrated that increased intestinal epithelial permeability in Crohn's disease (CD) may indeed precede clinical relapse by as much as 1 year and that unaffected first-degree relatives of $\mathrm{CD}$ patients may also have barrier dysfunction. In addition, it is well known that mucosal barrier-breaking substances, such as non-steroidal anti-inflammatory drugs (NSAIDs), may cause flare-ups in IBD patients. Finally, transgenic animal models have clearly demonstrated that a unique defect in the intestinal epithelial barrier is a sufficient trigger of the development of chronic gut inflammation. The recent advances in genotyping technology have greatly improved the knowledge base regarding genetic susceptibility for IBD and have revealed several IBD-associated single nucleotide polymorphisms (SNPs) in genes involved in intestinal barrier function.

In this chapter, we first describe the components of the normal intestinal barrier. Next, we focus on the different barrier anomalies found in IBD both at the genetic and molecular level. The current evidence for a role of these barrier disturbances in the inflammatory process is extensively discussed. As a final point, the different therapeutic strategies for protecting or restoring the barrier function of the gut during IBD are discussed. 


\section{Components of the normal intestinal barrier}

\subsection{The physical barrier is composed of a tightly linked intestinal epithelial cell layer and a mucus shield}

The surface lining of the intestine is composed of a single cell layer of tightly linked columnar epithelial cells. Intestinal epithelial cells are polarised, possessing an apical surface facing the lumen and a basolateral surface that is in direct contact with the immune compartment of the underlying lamina propria. The cells are tightly sealed by intercellular protein complexes consisting of tight junctions, adherens junctions and desmosomes (see 2.1.2), thus creating two physical compartments that separate the outside lumen from the inner host immune system. As such, these epithelial cells serve as a physical line of defence against harmful components passing the lumen, including foreign antigens, bacteria and the toxins they produce. Simultaneously, this barrier acts as a selective filter that permits the passage of essential dietary nutrients, electrolytes and water across the epithelial layer.

Selective transport through the intestinal epithelial membrane is accomplished in three ways: via the transcellular route, via the paracellular route and through microfold (M) cells (figure 1). The transcellular passage of amino acids, ions, sugars and short-chain fatty acids is performed by specific pumps and channels embedded in the cell membrane. This process is called transcytosis, and it involves the uptake of entities and their subsequent endosomal degradation. As such, the transport of intact proteins is limited, as they are degraded by the lysosomal system. The transcellular transport of bacteria and toxins is usually linked with mucosal inflammation. Paracellular transport refers to the passage of luminal materials through the space between the epithelial cells that is controlled by the intercellular junctional complexes. These complexes permit the diffusion of ions and solutes through the pores created by the protein structures and prevent the flux of larger entities such as microbes. Both the junctional pore size and the presence and activity of membrane pumps are highly regulated by such factors as cytokines and hormones, and these factors largely determine the passage and "leakiness" of the intestine. A final route of epithelial transport is mediated by $\mathrm{M}$ cells, which are typically located in overlying lymphoid aggregates called Peyer's patches. Unlike other epithelial cells, $M$ cells lack microvilli and a mucus coat and represent a "guarded gateway" for the entry of microbes that are quickly recognised by the underlying lymphoid tissue.

\subsubsection{Specialised epithelial cells}

The epithelial lining of the gastrointestinal tract is composed of self-renewing epithelial cells that are arranged as crypts and villus projections. Stem cells within the crypts give rise to different types of specialised epithelial cells that migrate to the tip of the villus, where they undergo programmed cell death. Paneth cells represent one exception because they remain in the crypts. In addition to its role in antigen trafficking, the intestinal epithelial lining represents an anatomic barrier (see 2.1.2). Moreover, epithelial cells play an active role in barrier protection; they produce mucus, regulate the composition of the mucus layer (see 2.1.3) and serve as antigen-presenting cells for the immune cells residing in the lamina propria (see 2.2). Several different types of specialised epithelial cells can be distinguished, each of which participates in specific barrier functions (Table 1). 


\begin{tabular}{|l|l|l|}
\hline \multicolumn{1}{|c|}{ Cell type } & \multicolumn{1}{|c|}{ Characteristics } & \multicolumn{1}{c|}{ Role in barrier function } \\
\hline Goblet cells & $\begin{array}{l}\text { Production and } \\
\text { release of mucus and } \\
\text { trefoil factors (see } \\
2.1 .3)\end{array}$ & $\begin{array}{l}\text { Formation of a semi-permeable mucus } \\
\text { layer preventing direct contact and } \\
\text { adhesion between microflora and } \\
\text { epithelial cells. } \\
\text { Increase in repair mechanisms and } \\
\text { tight junctions by trefoil factors. }\end{array}$ \\
\hline Paneth cells & $\begin{array}{l}\text { Production and } \\
\text { release of } \\
\text { antimicrobial } \\
\text { peptides }\end{array}$ & $\begin{array}{l}\text { Direct bactericidal or bacteriostatic } \\
\text { effects elicited by defensins, lysosyme } \\
\text { and phospholypase A2. Some } \\
\text { enveloped viruses and fungi can be } \\
\text { specifically lysed by these } \\
\text { antimicrobial peptides. }\end{array}$ \\
\hline Enteroendocrine cells & $\begin{array}{l}\text { Production and } \\
\text { release of serotonin }\end{array}$ & $\begin{array}{l}\text { Release of serotonin into the lamina } \\
\text { propria activates nerve fibres resulting } \\
\text { in the stimulation of mucin secretion } \\
\text { from goblet cells and passive water } \\
\text { efflux. }\end{array}$ \\
\hline M cells & $\begin{array}{l}\text { Selective uptake of } \\
\text { bacteria and antigens } \\
\text { from the lumen via } \\
\text { phagocytosis }\end{array}$ & $\begin{array}{l}\text { Controlled stimulation of the gut- } \\
\text { associated immune system. }\end{array}$ \\
\hline
\end{tabular}

Table 1. Main characteristics of the different epithelial cell types found in the gut and their role in intestinal barrier protection.

\subsubsection{Intercellular junction complexes}

Intestinal epithelial cells are sealed together by dynamic protein complexes composed of transmembrane proteins linked to the actin cytoskeleton through adaptor proteins. The intestinal epithelial cell lining in the gut is permanently self-renewing through the continuous migration of cells from the bottom of the crypt to the villus tip. To maintain the integrity of the epithelial barrier, intercellular complexes are rapidly assembled and disassembled without any dysfunction of the barrier function.

At the ultrastructural level, contacts between cells can be classified as tight junctions, adherens junctions or desmosomes. On the apical side of the epithelial monolayer, cells are attached to each other by means of tight junctions. These can be easily identified by electron microscopy because they leave no free space between two cells, in contrast to other junctional complexes in which cells are separated by 15 to $20 \mathrm{~nm}$. Tight junctions consist of three types of proteins: occludins, claudins and junctional adhesion molecules. These molecules are linked to the cytoskeleton by members of the zonula occludens (ZO) family. Below the tight junctions, cells are attached by adherens junctions composed of E-cadherin 


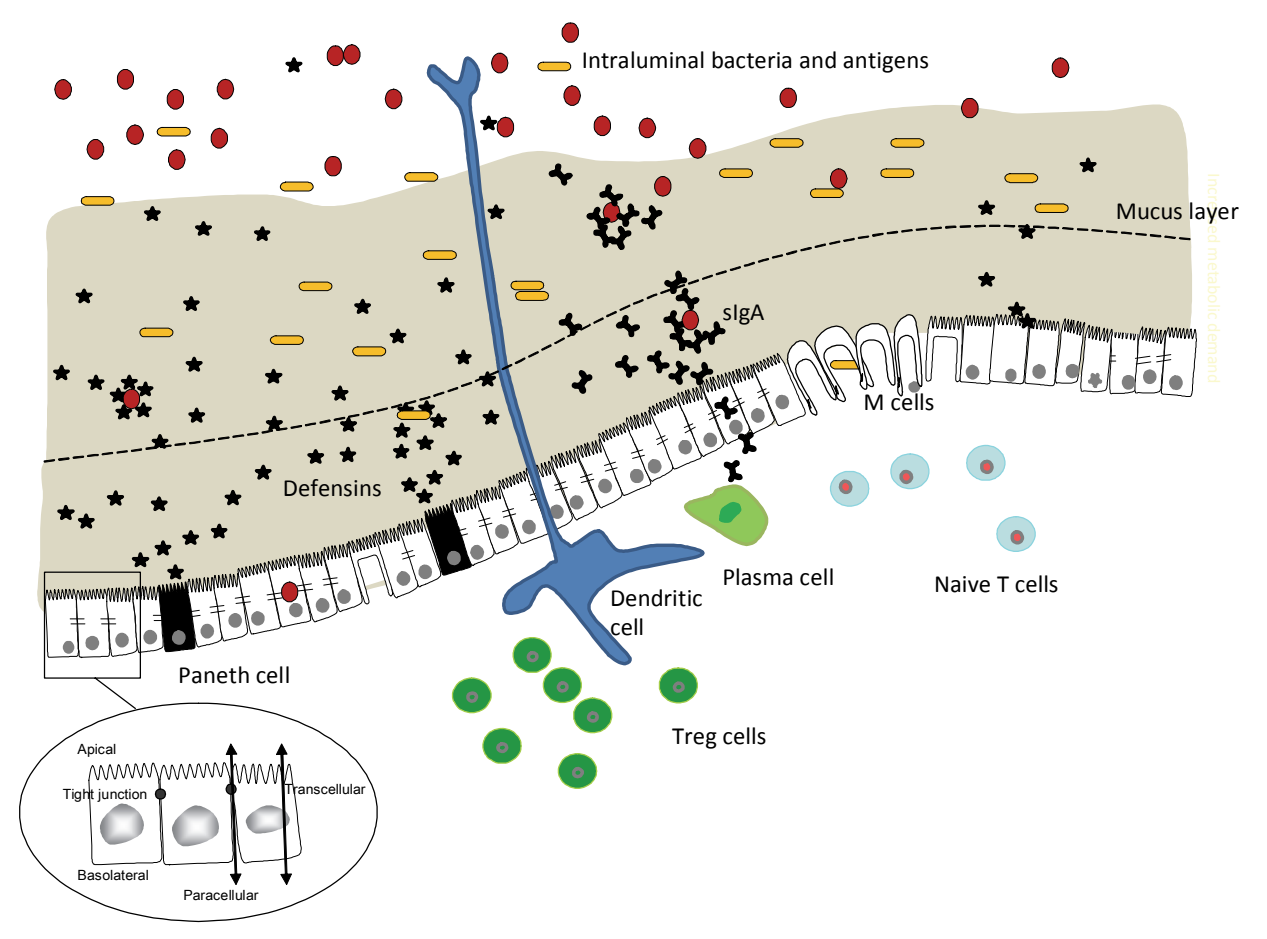

Fig. 1. Components of the normal intestinal barrier. Polarized intestinal epithelial cells provide a physical barrier between the outer luminal surfaces (apical) from the inner host immune tissues (basolateral). Highly selective transport across this barrier is accomplished by transcellular and paracellular routes and through $\mathrm{M}$ cells. A thick glycoprotein layer prevents direct contact between luminal bacteria and the epithelial cells. Further overgrowth of bacteria is prevented by the secretion of Paneth cell-derived antimicrobial peptides such as defensins and secreted $\operatorname{IgA}$ molecules ( $\operatorname{IgA}$ ) produced by plasma cells. Tolerance within the gut is mediated by the large number of tolerogenic dendritic cells that are able to sense the lumen for bacterial antigens, resulting in the development of regulatory $\mathrm{T}$ (Treg) cells.

molecules that are connected via catenin proteins. Finally, desmosomes reside at the basal part of the epithelial cell and provide anchoring points for keratin filaments that are attached to intracellular desmoplakin, which connects the cytoskeleton to proteins belonging to the cadherin family.

\subsubsection{The extracellular mucus shield}

Throughout the gastrointestinal tract, intestinal epithelial cells are covered on the apical side with a viscous glycoprotein layer, although the sites of $\mathrm{M}$ cells are an exception. This layer acts as a lubricant for the propulsion of gut contents and prevents direct contact between bacteria and epithelial cells, thus preventing inappropriate immune reactions. The mucus layer is porous, permitting the diffusion of macromolecules required for 
gastrointestinal absorption and digestion while impeding the invasion of bacterial-sized particles.

Four major components can be found in the complex mixture of the mucus barrier: secreted mucins and trefoil peptides (produced by goblet cells), antimicrobial peptides (produced by Paneth cells) and immunoglobulin A (IgA) molecules (produced by B cells residing in the lamina propria).

The mucus barrier consists of two layers: a thin, sterile inner layer and a bulkier outer mucus layer that contains bacteria. The outer mucus layer physically protects the underlying cells from luminal bacteria. However, another important function is that it represents a niche that houses the commensal bacteria colonising the gut, thereby maintaining a balanced microflora that facilitates digestion. This outer layer is a dynamic compartment that is continuously degraded by luminal flora and replaced by the underlying cells. The differentiation of goblet cells and the release of mucins and antimicrobial peptides are directly regulated by the microbial flora. In addition, pathogen recognition by innate mechanisms (see 2.2) leads to the production of cytokines, which consequently stimulate mucin release. High concentrations of antimicrobial peptides and secretory $\operatorname{IgA}$, which exert immune pressure on luminal bacteria, are found in the inner layer of the mucus barrier. Bile salts, which are produced mainly in the small intestine, greatly contribute to the suppression of bacterial growth in the mucus coat.

The mucus layer in the colon is thicker than that of the ileum. Furthermore, bile salt concentrations are much lower in the colon, whereas bacterial load and dwell time are higher.

\subsection{The innate defence system: Sensing microbe-associated molecular patterns}

When bacteria are able to break through the mucus barrier, either because of active pathogenic mechanisms or because the mucus layer is compromised, they reach the surface of epithelial cells. This triggers a rapid innate immune reaction mediated by TLRs and NOD-like receptors on the cell surface and inside epithelial cells, respectively. Rather than recognising specific antigens, these receptors discriminate self from non-self entities by recognising highly conserved molecular structures, the so-called microbe-associated molecular patterns. Examples include lipopolysaccharide (LPS), bacterial DNA, flagellin and peptidoglycan. Upon the binding of these ligands to their receptors, they recruit adaptor proteins, such as MyD88, inducing a signalling cascade that ends in the activation of nuclear factor kappa B (NFkB) and subsequent chemokine and pro-inflammatory cytokine expression, including tumour necrosis factor alpha (TNF $\alpha$, see 4.1).

At least 11 TLR homologues have been identified, each of which has the unique capacity to recognise a specific microbial pattern. The best-studied apical TLR is TLR4, which recognises LPS, a cell-wall constituent of Gram-negative bacteria. In contrast, TLR5 can bind bacterial flagella, and it is located on the basolateral side of epithelial cells, suggesting its involvement in the eradication of invading bacteria.

The NOD-like receptors are expressed exclusively within the cell. NOD1 and NOD2 have been widely studied, and each binds to a specific moiety of peptidoglycan, the main constituent of the bacterial cell wall of both Gram-negative and Gram-positive bacteria. After ligand binding, these receptors recruit the Rip2 protein, which in turn also leads to the activation of NFKB. 
The final outcome of innate signalling is the induction of pro-inflammatory cytokines and subsequent recruitment of phagocytes, which present bacterial antigens, leading to the activation of adaptive immune responses and clearance of the infection. Surprisingly, the deletion of TLR4 or MyD88 in mice leads to increased susceptibility to chemically induced colitis. Antibiotic therapy or germ-free cultivation of mice also results in a higher sensitivity to colitis. In addition, TLR4 signalling increases transepithelial resistance, indicating increased gut barrier function. It is clear that TLR and NOD signalling is important in maintaining a physiological state of immune activation to preserve intestinal homeostasis (see 2.3), and these signalling pathways are actively involved in repair mechanisms.

\subsection{The immunological barrier characterised by oral tolerance}

Once antigens invade the epithelial barrier, they are sensed by antigen-presenting cells (dendritic cells and resident macrophages), which prime naïve T cells in situ or after they migrate to the mesenteric lymph nodes. Activated $\mathrm{T}$ cells then differentiate into $\mathrm{T}$ helper type 1 (Th1), Th2, Th17 or regulatory $\mathrm{T}$ (Treg) cells and up-regulate specific gut-homing receptors ( $\alpha 4 \beta 7$ integrin and CCR9) to exert their functions at the site of infection. Whereas invasive pathogens can actively intrude on gut epithelial cells or induce their own phagocytosis via $M$ cells, non-invasive bacteria can enter dendritic cells because of their frequent sampling. In the gut, dendritic cells can access the lumen by opening the intercellular space through the expression of tight junction proteins without compromising epithelial barrier function.

The balance between responsiveness towards foreign antigens and unresponsiveness towards self-antigens is critical because any breakdown of these mechanisms can lead to autoimmunity or an inability to respond to harmful infections. An intriguing question, therefore, is how the gut, which contains an enormous amount of food components and bacteria and houses an elaborate network of lymphoid tissue, is not in a state of massive inflammation as a result of the constant triggering of innate immune responses, both from epithelial cells and the underlying lymphoid cells. The control of such responses is called oral tolerance, and this control precisely defines a healthy mucosal barrier. Immune homeostasis in the gut utilises innate signalling triggered mainly by the commensal microflora and provides an environment rich in Treg cells, which produce antiinflammatory cytokines such as interleukin 10 (IL10).

Oral tolerance is defined as the absence of a systemic immune response towards an antigen that has previously been encountered by the host. Thus, tolerance is an antigen-specific event. Together with anergy and apoptosis of antigen-specific $\mathrm{T}$ cells in the gut, the induction of antigen-specific Treg cells represents a method of actively inhibiting unnecessary inflammation. The key players in maintaining oral tolerance are the gutresident antigen-presenting cells, which produce regulatory and immunosuppressive cytokines and present antigens to naïve $\mathrm{T}$ cells. In particular, dendritic cells exert the greatest stimulatory effect on T cells, as they express high levels of MHC class II and costimulatory molecules. Emerging evidence suggests that dendritic cells in the gut are conditioned to a tolerogenic state, mediated by transforming growth factor beta 1 (TGF $\beta$ ), thymic stromal lymphopoietin (TSLP) and retinoic acid.

An important role exists for TGF $\beta$, a well-known immunosuppressive cytokine that is expressed abundantly in the gut. TGF $\beta$ inhibits the expression of the transcription factors T- 
bet and GATA-3, which are necessary for the differentiation of Th1 and Th2 cells, respectively. In addition, TGF $\beta$ stimulates cells to differentiate into Treg cells that express the transcription factor Foxp3, which in turn inhibits the expression of IL2 and results in reduced T cell proliferation. Finally, TGF $\beta$ induces Th17 cells that express the transcription factor ROR $\gamma \mathrm{T}$ and produce inflammatory cytokines IL17A, IL17F and IL22. Although these cells play an important role during infection, an excess of Th17 cells has been associated with tissue injury and chronic inflammation. The expansion of established Th17 cell populations is accomplished by IL23, a cytokine that alters intestinal homeostasis and that has been linked with susceptibility to IBD (see 3.2.5).

\subsection{Commensal bacteria}

Commensal bacteria are by definition non-pathogenic microbes (bacteria or yeast) that can colonise the intestinal environment and confer benefit to the host. Typically, these bacteria are known to play a role during food digestion. However, they also elicit considerable protective effects related to barrier function. A very important role of commensal bacteria relates to immunomodulation by promoting tolerogenic dendritic cell and Treg cell populations and inhibiting pro-inflammatory cytokine production as described above. For example, commensal bacteria can induce TSLP and TGF $\beta$ expression from epithelial cells. Some Lactobacillus species can increase mucus, IgA and defensin secretion, and several Bifidobacteria strains can directly inhibit adherence or invasion of specific pathogens into epithelial cells or inhibit their growth and destroy them. Finally, some commensals can strengthen epithelial junctional complexes by inducing the expression of host proteins in these complexes.

\section{Intestinal barrier dysfunction in IBD}

IBD is characterised by loss of controlled ion and water transport, which is a direct consequence of intestinal barrier dysfunction. Increased permeability in IBD is largely determined by tight junction deregulation and apoptosis of epithelial cells. Functionally, barrier loss is associated with uncontrolled immune responses because of increased bacterial translocation and high expression of inflammatory cytokines such as TNF $\alpha$ and IFN $\gamma$ (in CD) and IL13 (in the case of ulcerative colitis (UC)). In addition, recent advances in metagenomic sequencing have greatly aided in the characterisation and understanding of the composition of microflora, which was found to be significantly altered in IBD (see 4.2.2). Although barrier dysfunction has indisputably been recognised as a component of IBD pathogenesis, the question whether these defects are a primary cause or a consequence of the chronic disease process remains unanswered. Over the last decade, evidence has mounted that intestinal barrier impairment may be the primary driver of IBD.

\subsection{Clinical data}

The first clue that an intestinal mucosal barrier defect could be an early pathogenic event in IBD came from studies of first-degree healthy relatives of patients with CD. It was found that these relatives exhibit increased intestinal permeability compared to that in unrelated control subjects despite the absence of inflammation.

Complete mucosal healing appears to become the goal of future treatment for IBD. Preliminary data indicate that patients with complete mucosal healing have long-lasting and 
deep remission. Interestingly, $C D$ patients in remission with a persistent increase in intestinal permeability appear to have a greater risk of disease relapse.

NSAID use, stress and smoking are known risk factors for IBD relapse. All of these factors are known to cause intestinal barrier dysfunction. NSAIDs inhibit the synthesis of mucosaprotective prostaglandins, potentially leading to ulcerations and barrier breakdown. Prolonged psychological stress can induce ultrastructural epithelial abnormalities, increased bacterial translocation and low-grade inflammation. Finally, smoking is associated with an increase in apoptotic cell death of the intestinal epithelium, leading to conductive leaks in the gut barrier.

\subsection{Genetic and molecular evidence for intestinal barrier dysfunction in IBD}

Evidence suggests that increased gut permeability tends to be familial and is most likely genetically determined. Up to $40 \%$ of healthy first-degree relatives of patients with CD have been reported to exhibit increased intestinal permeability, compared to $5 \%$ in the control population.

Epidemiological evidence clearly indicates that CD and UC are related polygenic diseases, and this has been supported by results from genetic association studies. In 2009, the International IBD Genetics Consortium (www.ibdgenetics.org), a network of researchers investigating the genetics of IBD, was established. At present, 20,000 CD and a similar number of UC cases have been collected, together with equivalent numbers of populationbased healthy controls, from several countries in Europe, North America and Australia. This collaboration has resulted in three large-scale genome-wide association studies for CD and UC in which multiple risk loci have been identified (Anderson et al., 2011; Barrett et al., 2008; Imielinski et al., 2009). At the end of 2010, 99 replicated loci were found, and the genes within these loci have led to the discovery of new pathways involved in chronic gut inflammation. Many of these pathways involve intestinal barrier dysfunction, particular for UC (Barrett et al., 2009). Although the genome-wide scans have greatly improved our understanding of IBD pathogenesis, only an estimated $25 \%$ of the total genetic susceptibility can be explained by the currently identified disease-associated SNPs. In addition, due to linkage disequilibrium, the exact causative mutations remain to be identified. In the next section, the best-studied genetic associations involved in barrier function are discussed.

\subsubsection{Paneth cell dysfunction}

Paneth cells, which secrete antimicrobial factors, are found in the base of the crypts of the ileum. Many of the risk factors identified for IBD converge on Paneth cells and their function, including XBP1, NOD2, TCF4 and ATG16L1. The most intriguing finding of Paneth cell dysfunction was found in the intestinal epithelial-specific knockout of XBP1, a central regulator of endoplasmic reticulum (ER) stress. These mice spontaneously develop small bowel inflammation that markedly resembles some features observed in human IBD, such as crypt abscesses, leucocyte infiltration and ulcerations (Kaser et al., 2008). Among several identified abnormalities, including goblet cell depletion and accelerated cell renewal, Paneth cells were completely lost in these mice. These data demonstrate that XBP1 function, or ER stress in general, is critical for proper intestinal epithelial cell functioning. Obviously, cells with the highest protein synthesis burden (Paneth cells and goblet cells) suffer most from a deletion of ER stress mediators. In human IBD, increased ER stress can be detected in inflamed areas of the intestine (Bogaert et al., 2011). However, it remains to be investigated 
whether this is a primary dysfunction or secondary to inflammation. One of the arguments that ER stress abnormalities are a primary event in IBD arises from genetic studies. Linkage of the region that is in close proximity to XBP1 has been established previously, and deep sequencing of this gene resulted in the identification of polymorphisms associated with both CD and UC. Rare XBP1 variants can be found to a much higher degree in IBD patients than in healthy controls. In particular, four non-synonymous mutations are present only in IBD patients. Interestingly, these mutations have been linked with hampered ER stress responses in vitro.

Another well-characterised risk gene specifically for ileal CD is the innate immune receptor NOD2 (see 3.2.4). The finding that NOD2 is highly expressed in Paneth cells is particularly of interest because these cells provide host defence against microbes in the ileum, whereas they are not present in the normal colon. Lysozyme is an enzyme that breaks down bacterial cell wall components into muramyl dipeptide (MDP, GlcNAc-MurNAc), which is recognised by NOD2. Ileal expression of defensins was diminished in active regions in patients with NOD2 mutations, although this was not observed in the diseased colon. NOD2 acts as an inducer of defensins, an effect that is lost in patients carrying a homozygous mutation in NOD2. Rather, these patients exhibit defective epithelial defence, proliferation of bacteria and the potential loss of epithelial barrier function. Similarly, variants in TCF4 have been associated with ileal CD. TCF4 is a Wnt signalling transcription factor that plays a crucial role in Paneth cell development and directly activates defensin expression.

Finally, a coding variant in ATG16L1, a gene involved in autophagy, was identified in CD. Mice with only a partial loss of this gene and CD patients carrying the causal ATG16L1 variant exhibit structural defects in Paneth cell granules, the "protein storage" compartment of the cell (Cadwell et al., 2008). Proteins that are normally found within granules were visible in the cytoplasm. In addition, an altered transcriptional profile was found specifically in Paneth cells, consistent with a pro-inflammatory state. However, Atg1611-deficient mice do not develop spontaneous intestinal inflammation. Unexpectedly, NOD2 with MDP activation has recently been reported to induce autophagy via an ATG16L1-dependent pathway, and monocytes isolated from patients carrying a CD-associated NOD2 variant fail to induce autophagy in response to MDP and bacterial infection.

Taken together, the apparently divergent genetic findings point to a central impairment in Paneth cell function that is associated with IBD. Although the risk genes act at different levels, this can be explained by the fact that innate sensing, ER stress and autophagy are interconnected.

\subsubsection{Junctional complex genes}

A locus containing $C D H 1$, which encodes E-cadherin, a component of adherens junctions, was found to be associated with UC and possibly with CD. With this finding, the first genetic link between colorectal carcinoma and chronic colonic inflammation was established, with routine colonoscopic surveillance recommended for those at greatest risk. Some mutations have been associated with reduced levels of E-cadherin at the plasma membrane and an accumulation of cytoplasmic cadherin. Another interesting genetic candidate gene for UC is hepatocyte nuclear factor 4 alpha (HNF4A), a transcription factor involved in the regulation of genes that comprise cell-cell contacts that is aberrantly expressed in human IBD. The full knockout of this gene is lethal, with embryological anomalies in the intestine including reduced epithelial cell proliferation, loss of crypt formation and defective goblet cell maturation. The conditional knockout of HNF4A in the 
intestinal epithelium results in mice that develop increased epithelial permeability and alterations in mucin-associated genes. A strong association was found, specifically for UC, in a region containing laminin beta 1 (LAMB1), which encodes a subunit of the laminin family of proteins. Laminins are extracellular matrix glycoproteins that serve as major constituents of the basement membranes and play a key role in anchoring epithelial cells. Again, strongly reduced expression of laminins has been demonstrated in UC.

The inflammatory cytokines TNF $\alpha$ and interferon gamma $(I F N \gamma)$, both of which are highly expressed during active $C D$, significantly influence tight junction reorganisation, but the exact mode of action is incompletely understood. The pore-forming claudins (claudin 1, 3, 4, 5 and 8), which can form size- and charge-specific paracellular pores, are up-regulated in $\mathrm{CD}$, whereas occludin expression is reduced. Most likely, these changes occur as a consequence of inflammation, as they are not apparent during inactive disease. Nevertheless, determining how cytokines can modulate tight junction organisation is of great importance, as drugs targeting these cytokines have proven clinical efficacy in IBD.

\subsubsection{Genes involved in the constitution of the mucus shield}

A decrease of the number of goblet cells in IBD is associated with the reduced expression of mucins and a thinning of the mucus layer. Clinically, mucus diarrhoea is observed in UC, most likely because of the poor quality of the secreted mucus. Conversely, goblet cell dystrophy has been observed in CD with an increase in mucus production and decreases in antimicrobial peptides and tissue-regenerating trefoil factors. Moreover, colonic glycoprotein composition appears to be genetically determined, as it is more similar between monozygotic twins than between unrelated individuals.

In human intestinal tissue isolated from patients with IBD, disturbed expression of several mucin genes has been reported, and allelic variants in MUC2, MUC3A, MUC4 and MUC13 have been associated with IBD.

Striking evidence for a role of mucus secretion in the development of colitis arise from mice that carry mutations in muc2. Aberrant mucus biosynthesis in these mice results in the spontaneous development of colitis with striking resemblance to some aspects of human UC. Targeted mutations in muc2 lead to misfolding of the mucin 2 protein in goblet cells followed by massive ER stress induction accompanied by increased intestinal permeability and enhanced production of pro-inflammatory cytokines in the distal colon.

\subsubsection{Genes involved in innate immunity}

The IBD1 locus, originally mapped in 1996, represents the best-replicated region exhibiting linkage specifically to CD and not to UC. In 2001, two groups simultaneously identified NOD2 as the first susceptibility gene for CD (Hugot et al., 2001; Ogura et al., 2001). Hugot and colleagues employed the positional cloning strategy, whereas Ogura and co-workers identified NOD2 by the positional candidate gene approach.

The NOD2 protein belongs to the NOD1/Apaf-1 family, which comprises cytosolic proteins composed of an N-terminal caspase recruitment domain, a centrally located nucleotidebinding domain, and a C-terminal leucine-rich regulatory (LRR) domain. Three common SNPs in NOD2 were independently associated with CD: two missense mutations [R702W (c.2104C>T, SNP8) and G908R (c.2722G>C), SNP12], and one frameshift mutation [1007fs (c.3020insC, SNP13)] that truncates the protein by 30 amino acids. All three variants alter the C-terminal domain of the protein, and they are located within or close to the LRR domain, which is involved in ligand recognition. The heterozygous carrier frequency of these 
variants in CD ranges from 30 to $50 \%$, compared to frequencies of 3-15\% for homozygous or compound heterozygote. By comparison, $8-15 \%$ of healthy controls are heterozygous, and $0-1 \%$ of individuals carry a homozygous variant. The relative risk of developing CD if one of these variants is carried increases by a factor of 1.5 to 3 for heterozygous carriers, but the risk increases by a factor of 20 to 40 in homozygous or compound heterozygous individuals. Although this relative risk appears high, it must be stated that the absolute risk for developing $\mathrm{CD}$ is no more than 1 in 25 for homozygous carriers. This reduced penetrance can undoubtedly be explained by the requisite of environmental risk factors and/or additional genetic determinants. Clinically, $40 \%$ of healthy relatives of IBD patients who carry one of these SNPs have increased intestinal permeability.

The Nod1/Apaf-1 family of proteins displays striking similarity to a class of disease resistance $(\mathrm{R})$ proteins found in plants. Following specific recognition of pathogen products, these $\mathrm{R}$ proteins mediate a defence response associated with metabolic alterations and localised cell death at the site of pathogen invasion. The LRR domains of $R$ proteins are highly diverse and appear to be involved in the recognition of a wide array of pathogen components. Similar to the R proteins, NOD2 appears to play an important role in innate and acquired immunity as a sensor of bacterial components. Specifically, NOD2 participates in the signalling events triggered by host recognition of specific bacterial motifs and subsequently activates $\mathrm{NF \kappa B}$, the key mediator in the production of pro-inflammatory mediators. Naturally occurring peptidoglycan fragments were identified as the microbial motifs sensed by NOD2, specifically MDP, which is found in Gram-negative and Grampositive bacterial peptidoglycans.

The expression of NOD2 was first thought to be restricted to myeloid lineage cells, primarily monocytes. Moreover, its expression is enhanced by pro-inflammatory cytokines and

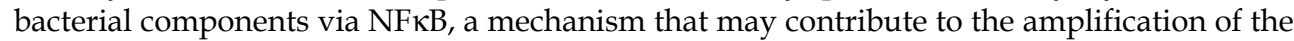
innate immune response. Consistent with this observation, elevated NOD2 expression has been detected in inflamed areas of colonic tissue of CD patients.

Although the function of NOD2 in bacterial sensing is widely accepted, its physiological function is less well understood. Consequently, the implications of the CD-associated mutations in disease onset and progression remain unclear. Several hypotheses have been postulated that involve both loss-of-function and gain-of-function mutations in NOD2, although the gain-of-function hypotheses have received criticism. The gain-of-function hypotheses evolved from NOD2 knockout and transgenic mice, but they are not consistent with the observations in humans. It is important to note that these hypotheses are not mutually exclusive and may be physiologically relevant in combination.

In vitro and ex vivo experiments have indicated that the three CD-associated polymorphisms actually decreased the activation of $\mathrm{NF \kappa B}$ and pro-inflammatory cytokine production, which are inconsistent with the observation that NFKB is up-regulated in patients. However, this might reflect a lack of primary innate immune triggering in response to bacterial invasion. As the three CD-associated variants of NOD2 are located in or near the LRR, it was suggested that bacterial sensing is impaired, thereby explaining the susceptibility to disease. Consequently, the clearing of bacterial products is inefficient, which may lead to a secondary, compensatory activation of NFKB independent of NOD2. Notably, peripheral blood mononuclear cells from individuals homozygous for the major disease-associated SNP13 mutation did not respond to synthetic MDP. These cells also exhibit defective proinflammatory cytokine release after stimulation with MDP. Mononuclear cells isolated from CD patients carrying NOD2 polymorphisms produced significantly less IL1 $\beta$, IL6 and IL10 
after stimulation with adherent-invasive E. coli LF82 in a gene-dose effect. This was the first study in which aberrations were found in heterozygous carriers of SNP8 and SNP12 NOD2 mutations, which represent the largest group of patients.

In human monocyte-derived dendritic cell cultures, NOD2 agonists synergistically induce IL12 production in combination with TLR3, TLR4 and TLR9 agonists to induce Th1-lineage immune responses. This synergistic effect was lost in patients carrying a mutant NOD2 protein. The inflammatory phenotype of CD is difficult to reconcile with decreases in TNF $\alpha$ and IL12 levels. However, it was recently demonstrated that the synergistic induction of IL10 in response to MDP and TLR stimuli was lost in NOD2 mutant monocyte-derived dendritic cells. IL10 is crucially involved in the down-regulation of the inflammatory process. It was thus postulated that IL10-mediated immune suppression is impaired, and the counter-effect for pro-inflammatory cytokines is lost, thereby contributing to chronic inflammation in CD.

Incubation of normal murine macrophages with MDP was demonstrated to suppress IL12 secretion induced by stimulation with TLR2 ligands. This suppression did not occur in cells lacking NOD2 or in cells expressing a mutant form of NOD2 during transfection experiments. Once secreted, IL12 promotes IFN $\gamma$ production and the growth and differentiation of Th1 cells. A major concern, however, is the reproducibility of these results. Furthermore, NOD2 and TLR2 stimulation of human mononuclear cells isolated from patients with the $1007 \mathrm{fs}$ mutation led to a loss of the synergistic induction of proinflammatory cytokines, which is also inconsistent with a TLR2 inhibitory function of NOD2.

LPS, a cell wall component of Gram-negative bacteria, is a major inducer of inflammation, and its signalling is mediated through the cell-surface receptor TLR4. During intestinal inflammation, TLR4 is up-regulated on epithelial cells, macrophages and dendritic cells, thus providing a first line of defence against enteric Gram-negative bacteria. An association between a polymorphism in the LRR region of TLR4 has been reported within a Dutch CD and UC cohort. Allele frequencies of $11 \%$ were found in CD patients, versus $5 \%$ in healthy controls. The association was replicated twice but could not be reproduced in three other studies. This mutation was previously linked to decreased bronchial responsiveness to LPS and impaired LPS signalling. However, no functional defect, e.g., cytokine release or LPS recognition, has been attributed to heterozygous carriers among $C D$ patients. However, TLR4 is generally up-regulated on intestinal epithelial cells of patients with IBD, contributing to prolonged and increased responsiveness to normal luminal bacteria.

\subsubsection{Genes involved in oral tolerance}

IBDs are characterised by a loss of tolerance towards commensal bacterial flora. Animal models of enteritis, such as the IL10 knockout model, do not develop disease when they are housed in a germ-free environment. Likewise, diversion of the faecal stream in IBD patients can stop the disease until the faecal stream is restored.

Anti-inflammatory cytokines, such as IL10, play a crucial role in maintaining tolerance. Deletion of the gene encoding IL10 or its receptor (IL10R) in mice results in spontaneous small intestinal inflammation. IL10R mutations have been found in CD. These mutations lead to inefficient immunosuppression via IL10.

Treg cells are important for the maintenance of intestinal self-tolerance. Concentrations of TSLP, a major inducer of Treg development, are reduced in patients with IBD. Although Treg numbers increase during active IBD, they are significantly lower when compared to 
non-IBD inflammatory conditions, such as diverticulitis. The addition of Treg cells in established colitis in mice results in complete remission of intestinal inflammation.

The first large genome-scan for CD resulted in the identification of multiple genes involved in IL23/Th17 signalling (Barrett et al., 2008), with CD-associated SNPs in CCR6, STAT3, JAK2, IL23R and IL12B.

\subsection{Animal data}

Additional evidence that barrier dysfunction may be the primum movens in IBD can be derived from animal studies. One of the earliest histological signs of dextran sodium sulphate (DSS)-induced colitis, a commonly used mouse model of UC, is increased apoptosis in colonocytes. This finding occurs before any histological sign of inflammation, such as an influx of polymorphonuclear cells in the colonic mucosa. A similar finding has been observed in the SAMP1/Yit and IL10 gene-deficient mouse models of CD. These mice both exhibit a disturbance of the intestinal epithelial integrity prior to the onset of inflammation.

Some genetically engineered mice with a primary defect in a component of the normal intestinal mucosal barrier ( $M u c 2, H n f 4 a$ gene-knockout mice) spontaneously develop chronic intestinal inflammation, suggesting that intestinal barrier disruption may be a primary and sufficient trigger of IBD.

\section{Strategies to defend or restore the gut barrier in IBD}

\subsection{Currently used therapies}

Little is known about the barrier-protective actions of the currently used drugs in IBD treatment.

Preparations of 5-aminosalicylic acid (5-ASA) are mainly used in the treatment of mild to moderate UC. The exact mechanism of action of 5-ASA is incompletely known. In the DSSinduced colitis model, mesalazine both reduces colonic inflammation and permeability. It has been claimed that part of the mode of action of 5-ASA might rely on a reduction of IFN $\gamma$-induced epithelial barrier dysfunction during inflammation.

Although sometimes used for maintenance of remission in patients with $\mathrm{CD}$, methotrexate appears instead to promote barrier dysfunction by promoting the production of reactive oxygen species and by altering ZO-1. Hence, it can be speculated that the anti-inflammatory actions of methotrexate overrule its negative effects on intestinal barrier function in patients with CD.

The introduction of anti-TNF agents was a breakthrough in the management of IBD. These biologics can rapidly induce remission and mucosal healing in both CD and UC patients. Anti-TNF agents share the ability to rapidly restore intestinal mucosal barrier function (within 2 weeks after a single infusion), which has been attributed to their strong anti-apoptotic effect on the gut epithelium rather than an effect on tight junction proteins.

\subsection{Experimental therapies}

Compounds with strong protective effects on the gut barrier often have spectacular efficacy in experimental gut inflammation, making them attractive candidates for the treatment of IBD. In this part, we give an overview of the most promising barrier-protecting molecules that might enter clinical practice in the future. 


\subsubsection{Prolyl hydroxylase-inhibiting compounds}

Active inflammation is associated with low levels of oxygen and nutrients. Severe intestinal mucosal hypoxia has been clearly demonstrated in both experimental colitis and human IBD. The intestinal epithelial cells lining the gut lumen are particularly prone to these decreased oxygen levels during inflammation because of their anatomic position, which is relatively far from the richly vascularised sub-epithelial mucosa (figure 2). Mucosal hypoxia will lead to epithelial inflammation and barrier dysfunction by stimulating the release of pro-inflammatory cytokines. Animals exposed to full-body hypoxia display increased colonic permeability and an increase in myeloperoxidase levels, which is a marker for neutrophil accumulation.

The inhibition of cellular PHDs during hypoxia is an endogeneous adaptive system of the enterocytes aimed at protecting against hypoxia-induced cell death. As a result of PHD inactivation, both hypoxia-inducible factor 1 (HIF-1) and NFKB are activated. HIF-1 is a transcription factor of many genes involved in angiogenesis, metabolism and barrier preservation. Mice with a conditional knockout of HIF-1 in the intestinal epithelium are more susceptible to chemically induced colitis. NFKB is considered a pro-inflammatory transcription factor. However, it has recently been recognised that $\mathrm{NF \kappa B}$ also has strong anti-apoptotic effects on the gut epithelium. Mice lacking NEMO, an important and essential positive regulator of $\mathrm{NF \kappa B}$, exhibit severe spontaneous colitis.

PHD-inhibiting compounds have a very strong protective action in murine models of colitis and in a murine model of Crohn's ileitis. A strong inhibitory effect on intestinal epithelial apoptosis appears to be an important mode of action. Future studies must address the feasibility and safety of these molecules for human use (Hindryckx et al., 2011a)

\subsubsection{Probiotics}

The normal intestinal microflora plays an important role in maintaining intestinal health. These microflora protect against pathogens and maintain epithelial barrier integrity. A microbial imbalance in the gut (termed "dysbiosis"), with a relatively low proportion of beneficial flora and a relatively high proportion of potentially harmful flora, has been associated with IBD. For example, some patients with CD have remarkably reduced levels of Faecalibacterium prausnitzii, a butyrate-producing commensal bacterium of the gut. Low ileal levels of this bacterial species are associated with a higher risk of postoperative recurrence of ileal CD. In addition, oral administration of Faecalibacterium prausnitzii or its supernatant appears to correct the dysbiosis in experimental colitis and strongly reduces the severity of inflammation. Butyrate functions as a potent anti-inflammatory factor through the inhibition of NFKB activation and the increased production of mucins, antimicrobial peptides and tight junction proteins. Therefore, the selection of probiotic strains from the Clostridium IV cluster of bacteria that can locally produce butyrate is under investigation (Van Immerseel et al., 2010)

Preliminary data on the use of probiotics in IBD patients to induce and maintain remission are promising, although the results appear to depend on the exact type of probiotic employed. For example, the addition of Saccharomyces boulardii to baseline therapy in patients with $\mathrm{CD}$ in remission may reduce the intestinal permeability and reduce the risk of relapse, although this was not observed for Lactobacillus casei GG.

Several probiotics have been successfully tested in the preclinical IBD setting, but they remain to be examined in human disease. For example, Lactobacillus plantarum has been 
demonstrated to ameliorate colonic epithelial barrier dysfunction and prevent colitis in IL10 knockout mice.

A careful selection of probiotic agents, combined with more convincing efficacy in large clinical trials, will determine whether some probiotics can be used to treat IBD in clinical practice.

\subsubsection{Flavonoids}

Flavonoids, a class of plant secondary metabolites, have long been recognised to exhibit some anti-inflammatory properties that may have potential applicability in IBD. For example, the flavonoid quercetin ameliorates experimental colitis in rats. In vitro studies have demonstrated that quercetin improves the barrier function of the gut by changing the expression and distribution of several tight junction proteins.

Curcumin, a flavonoid extract from the spice turmeric, has been demonstrated to protect the mucosal barrier during rat enteritis. In a double-blind, multicentre trial of UC patients, the combination of curcumin and 5-ASA was superior in the prevention of disease relapse to 5ASA alone.

Although evidence for a beneficial role in human IBD is still scarce, the available data on flavonoids suggest that it could be an effective and safe supplement to conventional IBD treatments.

\subsubsection{Phosphatidylcholine}

Phosphatidylcholine is a major phospholipid component of cell membranes. Insufficient phosphatidylcholine in the colonic mucus may lead to impaired phospholipid barrier function in UC, resulting in exposure to colonic commensal bacteria and mucosal inflammation. Exogenously administered phosphatidylcholine has anti-inflammatory properties in murine models of colitis.

Phase IIa/b clinical trials have demonstrated that delayed-release phosphatidylcholine can induce clinical improvement and even remission in UC patients.

\subsubsection{Oxygenated perfluorodecalin}

PFD is a member of the perfluorochemical (PFC) family of chemicals, which are highdensity inert liquids with a remarkable capacity to dissolve high amounts of oxygen. These molecules can be used as powerful oxygen carriers and releasers into ischemic tissues. Topical administration of oxygenated PFCs has already been successfully used to treat difficult-to-treat chronic wounds, such as burns and diabetic ulcers. Intrarectal administration of oxygenated PFD both prevents and cures experimental colitis (Hindryckx et al., 2011b). The mechanism of action largely relies on a protective effect on colonic epithelial barrier function during inflammation. Due to its high density, PFD covers the colonic mucosa with an impermeable film, thereby preventing the influx of luminal antigens through the damaged colonic mucosa. In addition, the oxygen released by oxygenated PFD prevents apoptosis and stimulates proliferation of the colonocytes during inflammatory hypoxia (figure 2).

No study on the use of oxygenated PFCs in human IBD has been conducted thus far. However, intrarectal administration of oxygenated PFCs is a typical example of a barrierpromoting treatment that could be a very attractive strategy to heal therapy-resistant distal IBD or pouchitis. 


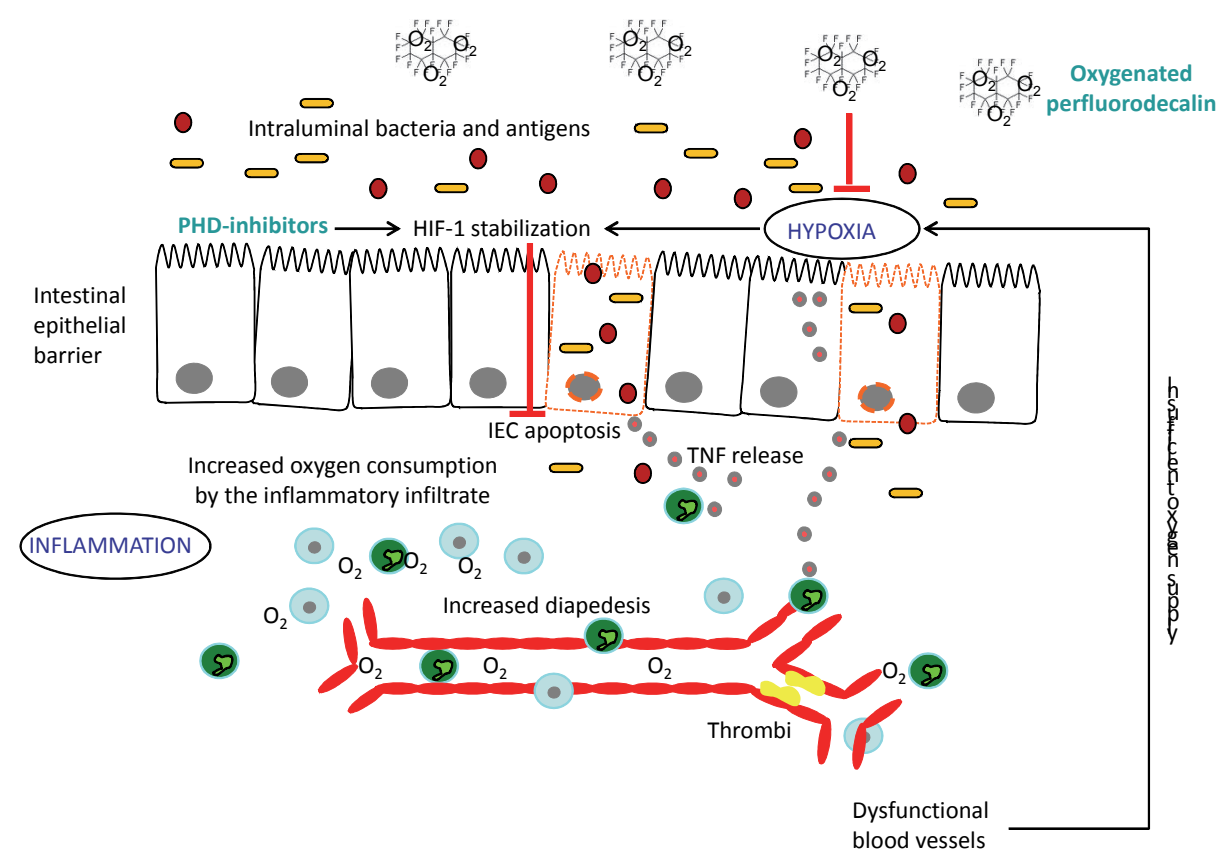

Fig. 2. Targeting inflammatory hypoxia in IBD to protect the gut barrier during inflammation. IBD is characterized by dysfunctional blood increased oxygen consumption by the active inflammatory infiltratea disturbed barrier function of the gut. Severe mucosal hypoxia during active IBD may lead to IEC death and further disruption of the gut barrier. Moreover, both hypoxia and inflammation stimulate the release of TNF- $\alpha$, which also has a pro-apoptotic action on the gut epithelium. Prolyl hydroxylase- (PHD-) inhibiting compounds mimick hypoxia and stabilize hypoxia-inducible factor-1 (HIF-1), which is a major transcription factor for cell survival and barrier-protection. Intra-luminal administration of oxygenated perfluorodecalin $\left(\mathrm{O}_{2}-\mathrm{PFD}\right)$ directly delivers $\mathrm{O}_{2}$ to the intestinal epithelium and suppresses inflammatory cytokines such as TNF- $\alpha$.

Both PHD-inhibitors and $\mathrm{O}_{2}$-PFD have been succesfully used in animal models of IBD.

\subsubsection{Growth factors}

Several observations have suggested that therapeutic growth factor administration attenuates the mucosal barrier function defect in CD.

Epidermal growth factor (EGF) plays a key role in the healing response of the gut. In a small but randomised placebo-controlled trial, EGF enemas were superior to placebo in inducing remission at week 2 in mesalazine-treated patients with distal UC.

Keratinocyte growth factor administration improved mucosal healing in murine colitis models but failed in a placebo-controlled trial in patients with active UC.

In addition to improving the microbicidal activity of phagocytic cells, granulocyte-macrophage colony-stimulating factor (GM-CSF) also stimulates the proliferation of colonic epithelial cells. In a multicentre, randomised, controlled clinical trial using patients with active $C D$, 
sargramostim (a recombinant version of GM-CSF) was superior to placebo in terms of disease remission and quality of life improvement.

Transforming growth factors (TGF $\alpha$, TGF $\beta$ ) play an important role in mucosal defence and repair. Both TGF subtypes have been demonstrated to be crucially protective in murine colitis. Preliminary maintenance studies with a polymeric diet rich in TGF $\beta$ have been performed in both paediatric and adult CD patients with satisfactory results.

Although the supplementation of some growth factors appears promising for treating IBD, concern exists regarding potential carcinogenic action, which should be addressed.

\subsubsection{Stem cells}

Stem cells (SCs) have pluripotent potential and can differentiate into every type of cell, including intestinal epithelial cells. This unique capacity may allow SCs to restore the intestinal epithelium as well as the immune balance in IBD. Currently, both haematopoietic SC transplantation and mesenchymal SC transplantation in IBD patients are under evaluation in phase III clinical trials.

\section{Conclusions}

IBDs are chronic, relapsing inflammatory conditions of the digestive tract, with an incompletely known multifactorial aetiology. Clinical, experimental and genetic data suggest that intestinal mucosal barrier dysfunction is a hallmark of IBD. The occurrence of increased permeability in healthy first-degree relatives of IBD patients and its predictive value of clinical relapse suggests that these events are of primary origin or are at least very early events in the pathogenesis of IBD. Therefore, restoration of the barrier integrity may be a highly effective treatment strategy to promote the recovery of the barrier function and help to alleviate inflammation.

\section{References}

Anderson C. A., Boucher G., Lees C. W., Franke A. et al. (2011). Meta-analysis identifies 29 additional ulcerative colitis risk loci, increasing the number of confirmed associations to 47. Nat Genet, Vol.43, No.3, pp. 246-52, ISSN 1546-1718

Barrett J. C., Hansoul S., Nicolae D. L., Cho J. H. et al. (2008). Genome-wide association defines more than 30 distinct susceptibility loci for Crohn's disease. Nat Genet, Vol.40, No.8, pp. 955-62, ISSN 1546-1718

Barrett J. C., Lee J. C., Lees C. W., Prescott N. J. et al. (2009). Genome-wide association study of ulcerative colitis identifies three new susceptibility loci, including the HNF4A region. Nat Genet, Vol.41, No.12, pp. 1330-4, ISSN 1546-1718

Bogaert S., De Vos M. Olievier K., Peeters H. et al. (2011). Involvement of endoplasmic reticulum stress in inflammatory bowel disease: A different implication for colonic and ileal disease? PloS One, E25589. ISSN 1932-6203

Cadwell K., Liu J. Y., Brown S. L., Miyoshi H. et al. (2008). A key role for autophagy and the autophagy gene Atg1611 in mouse and human intestinal Paneth cells. Nature, Vol.456, No.7219, pp. 259-63, ISSN 1476-4687 
Hindryckx P., Laukens D. \& De Vos M. (2011a). Boosting the hypoxia-induced adaptive response in inflammatory bowel disease: A novel concept of treatment. Inflamm Bowel Dis, Vol.17, No.9, pp. 2019-22. ISSN 1536-4844

Hindryckx P., Devisscher L., Laukens D., Venken K. et al. (2011b). Intrarectal administration of oxygenated perfluorodecalin promotes healing of murine colitis by targeting inflammatory hypoxia. Lab Invest, Vol.91, No.9, pp. 1266-76 ISSN 1530-0307

Hugot J. P., Chamaillard M., Zouali H., Lesage S. et al. (2001). Association of NOD2 leucinerich repeat variants with susceptibility to Crohn's disease. Nature, Vol.411, No.6837, pp. 599-603, ISSN 0028-0836

Imielinski M., Baldassano R. N., Griffiths A., Russell R. K. et al. (2009). Common variants at five new loci associated with early-onset inflammatory bowel disease. Nat Genet, Vol.41, No.12, pp. 1335-40, ISSN 1546-1718

Kaser A., Lee A. H., Franke A., Glickman J. N. et al. (2008). XBP1 links ER stress to intestinal inflammation and confers genetic risk for human inflammatory bowel disease. Cell, Vol.134, No.5, pp. 743-56, ISSN 1097-4172

Ogura Y., Bonen D. K., Inohara N., Nicolae D. L. et al. (2001). A frameshift mutation in NOD2 associated with susceptibility to Crohn's disease. Nature, Vol.411, No.6837, pp. 603-6, ISSN 0028-0836

Van Immerseel F., Ducatelle R., De Vos M., Boon N. et al. (2010) Butyric acid-producing anaerobic bacteria as a novel probiotic treatment approach for inflammatory bowel disease. J Med Microbiol, Vol.59, No.2, pp. 141-3, ISSN 1473-5644 


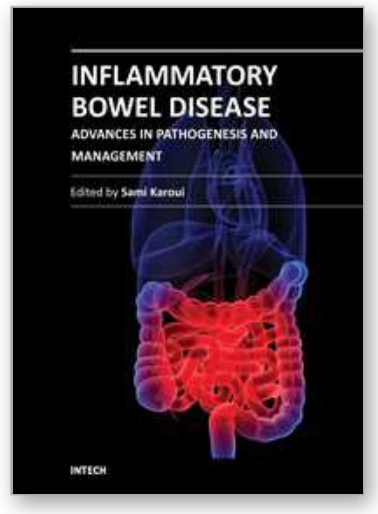

\author{
Inflammatory Bowel Disease - Advances in Pathogenesis and \\ Management \\ Edited by Dr. Sami Karoui
}

ISBN 978-953-307-891-5

Hard cover, 332 pages

Publisher InTech

Published online 27, January, 2012

Published in print edition January, 2012

This book is dedicated to inflammatory bowel disease, and the authors discuss the advances in the pathogenesis of inflammatory bowel disease, as well as several new parameters involved in the etiopathogeny of Crohn's disease and ulcerative colitis, such as intestinal barrier dysfunction and the roles of TH 17 cells and IL 17 in the immune response in inflammatory bowel disease. The book also focuses on several relevant clinical points, such as pregnancy during inflammatory bowel disease and the health-related quality of life as an end point of the different treatments of the diseases. Finally, advances in management of patients with inflammatory bowel disease are discussed, especially in a complete review of the recent literature.

\title{
How to reference
}

In order to correctly reference this scholarly work, feel free to copy and paste the following:

Pieter Hindryckx and Debby Laukens (2012). Intestinal Barrier Dysfunction: The Primary Driver of IBD?, Inflammatory Bowel Disease - Advances in Pathogenesis and Management, Dr. Sami Karoui (Ed.), ISBN: 978953-307-891-5, InTech, Available from: http://www.intechopen.com/books/inflammatory-bowel-diseaseadvances-in-pathogenesis-and-management/intestinal-barrier-dysfunction-the-primary-driver-of-ibd-

\section{INTECH}

open science | open minds

\author{
InTech Europe \\ University Campus STeP Ri \\ Slavka Krautzeka 83/A \\ 51000 Rijeka, Croatia \\ Phone: +385 (51) 770447 \\ Fax: +385 (51) 686166 \\ www.intechopen.com
}

\author{
InTech China \\ Unit 405, Office Block, Hotel Equatorial Shanghai \\ No.65, Yan An Road (West), Shanghai, 200040, China \\ 中国上海市延安西路65号上海国际贵都大饭店办公楼 405 单元 \\ Phone: +86-21-62489820 \\ Fax: +86-21-62489821
}


(C) 2012 The Author(s). Licensee IntechOpen. This is an open access article distributed under the terms of the Creative Commons Attribution 3.0 License, which permits unrestricted use, distribution, and reproduction in any medium, provided the original work is properly cited. 